\title{
RESPOSTA DA CULTURA DO FEIJOEIRO À ADUBAÇÃO NITROGENADA E À INOCULAÇÃO COM RIZÓBIO(1)
}

\author{
Rodrigo de Pelegrin ${ }^{(2)}$, Fábio Martins Mercante ${ }^{(3)}$, Ilda Miyuki \\ Nakase Otsubo ${ }^{(2)} \&$ Auro Akio Otsubo ${ }^{(3)}$
}

\begin{abstract}
RESUMO
O manejo adequado da adubação nitrogenada representa uma das principais dificuldades da cultura do feijoeiro (Phaseolus vulgaris L.). No entanto, a fixação biológica de nitrogênio é uma fonte alternativa de suprimento deste nutriente à cultura. $O$ presente estudo teve como objetivo avaliar a resposta do feijoeiro à inoculação com rizóbio e ao parcelamento de fertilizante nitrogenado em termos de nodulação das plantas e produtividade de grãos da cultura, bem como a viabilidade econômica da aplicação de fertilizante nitrogenado e, ou, inoculação com rizóbio em feijoeiro. $O$ experimento foi conduzido num Latossolo Vermelho distroférrico, em Dourados, MS. O delineamento experimental utilizado foi o de blocos casualizados, com seis repetições. Os sete tratamentos consistiram da aplicação parcelada de fertilizante nitrogenado em diferentes doses $(0,20,40,80 \mathrm{e}$ $160 \mathrm{~kg} \mathrm{ha}^{-1}$ de $\mathrm{N}$ como ureia) na cultura do feijoeiro, cv. Pérola, além de controles com inoculação de Rhizobium tropici combinada ou não com aplicação de $20 \mathrm{~kg} \mathrm{ha}^{-1}$ de N. A análise econômica foi efetuada considerando os custos da ureia e sua aplicação a lanço, além do custo do inoculante; os demais custos não foram considerados, por não haver diferença entre os diferentes tratamentos. Foram obtidos o acréscimo de produtividade, o custo de produção, o acréscimo da receita bruta e o acréscimo da receita líquida dos tratamentos, em relação à testemunha sem inoculação e sem adubação nitrogenada. Embora a nodulação das plantas não tenha sido alterada pelos tratamentos, verificou-se tendência de redução conforme o aumento da dose de $\mathrm{N}$ aplicada. A inoculação com rizóbio selecionado promoveu rendimentos de grãos de feijoeiros equivalentes à aplicação de $80 \mathrm{~kg} \mathrm{ha}^{-1} \mathrm{de} \mathrm{N}$. Quando acrescida da adubação com $20 \mathrm{~kg} \mathrm{ha}^{-1}$ de $\mathrm{N}$ no plantio, a inoculação com rizóbio propiciou acréscimo de receita líquida semelhante à aplicação de $160 \mathrm{~kg} \mathrm{ha}^{-1}$ de $\mathrm{N}$ e superior ao tratamento com a adubação de $20 \mathrm{~kg} \mathrm{ha}^{-1}$ de $\mathrm{N}$ sem
\end{abstract}

\footnotetext{
${ }^{(1)}$ Recebido para publicação em junho de 2008 e aprovado em janeiro de 2009.

(2) Graduado em Agronomia pela FAD/UNIDERP (Faculdade de Dourados / Universidade para o Desenvolvimento do Estado e da Região do Pantanal), Embrapa Agropecuária Oeste. Av. Presidente Vargas 1775, Vila Progresso, 79830-090 Dourados (MS). Trabalho conduzido como bolsista do CNPq. E-mails: rdpelegrin@hotmail.com; ilda@cpao.embrapa.br

(3) Pesquisador da Embrapa Agropecuária Oeste, Caixa Postal 661, CEP 79804-970 Dourados (MS). E-mails: mercante@cpao.embrapa.br; auro@cpao.embrapa.br
} 
inoculação, evidenciando a sua importância para obtenção de maior rentabilidade na cultura do feijoeiro.

Termos de indexação: feijão comum, Rhizobium tropici, análise econômica.

\title{
SUMMARY: RESPONSE OF COMMON BEAN CROP TO NITROGEN FERTILIZATION AND RHIZOBIUM INOCULATION
}

\begin{abstract}
Adequate management of nitrogen fertilizer is one of the main difficulties on common bean (Phaseolus vulgaris L.) crop. However, the biological $N_{2}$ fixation is an alternative source of $N$ supply to this crop. This study had the purpose to evaluate the response of common bean to rhizobium inoculation and split application of $N$ fertilizer in terms of plant nodulation and grain yield. Besides, this study aimed to evaluate the economic viability of using $N$ fertilizer application and /or rhizobium inoculation on the plants. The experiment was carried out on a typic Haplustox in Dourados, Mato Grosso do Sul State. The experimental design was a completely randomized block with six replications. The seven treatments consisted of different levels of $N$ fertilizer $\left(0,20,40,80\right.$, and $160 \mathrm{~kg} \mathrm{ha}^{-1} \mathrm{~N}$ as urea) applied to common bean, cultivar Pérola, and control treatments with R. tropici inoculation with or without application of $20 \mathrm{~kg} \mathrm{ha}-1$ N. In the economic analysis, the cost of urea, broadcast application, and cost of inoculant were taken into account. No other costs were considered since the treatments did not differ. Increases in grain yield, production cost, net and gross income, in relation to the control without inoculation and without nitrogen were obtained. The effect of inoculation of selected rhizobia on bean grain yield was similar to the application of $80 \mathrm{~kg} \mathrm{ha}^{-1} \mathrm{~N}$. When supplied with $20 \mathrm{~kg} \mathrm{ha}^{-1} \mathrm{~N}$ at sowing, rhizobium inoculation increased grain yield and net income similarly to the application of $160 \mathrm{~kg} \mathrm{ha}^{-1} \mathrm{~N}$ and more than the treatment of $20 \mathrm{~kg} \mathrm{ha}-1 \mathrm{~N}$ without inoculant, which demonstrates the importance of the inoculant for an enhanced crop profitability.
\end{abstract}

Index terms: common bean, Rhizobium tropici, economic analysis.

\section{INTRODUÇÃO}

No Brasil, a importância social e econômica da cultura do feijoeiro é evidenciada, principalmente, por representar uma importante fonte proteica na dieta alimentar da população e pelo contingente de pequenos produtores envolvidos na sua produção, embora tenha havido nos últimos anos crescente interesse de produtores de outras classes do agronegócio, adotando técnicas avançadas, incluindo a irrigação e a colheita mecanizada (Yokoyama, 2002). A produtividade média brasileira na safra 2005/06 ficou em torno de $822 \mathrm{~kg} \mathrm{ha}^{-1}$, enquanto no Estado de Mato Grosso do Sul atingiu $945 \mathrm{~kg} \mathrm{ha}^{-1}$, nessa mesma safra (CONAB, 2008).

Entre os principais fatores limitantes da produtividade da cultura do feijoeiro no País, destacam-se aqueles relacionados ao baixo nível técnico empregado pelos produtores e ao cultivo do feijoeiro em solos de baixa fertilidade, especialmente pobres em N (Caballero et al., 1985; Mercante et al., 1999).

As principais fontes de $\mathrm{N}$ para a cultura do feijoeiro são o solo, por meio da decomposição da matéria orgânica, a aplicação de adubos nitrogenados e a fixação biológica de $\mathrm{N}_{2}$ atmosférico, pela associação do feijoeiro com bactérias do grupo dos rizóbios (Hungria et al., 1997; Mercante et al., 1999). Além do elevado custo econômico, o uso de adubos nitrogenados em solos tropicais tem ainda um custo ecológico adicional. Considera-se que as perdas de adubos nitrogenados aplicados estão em torno de $50 \%$, sendo ocasionadas principalmente por lixiviação, na forma de nitrato e escorrimento superficial, provocado pela água das chuvas e, ou, irrigação (Straliotto et al., 2002). O N perdido nesse processo é altamente poluente e, uma vez carreado para o lençol freático, provoca a contaminação dos aquíferos subterrâneos, rios e lagos. Outras perdas de $\mathrm{N}$ aplicado ocorrem nas formas gasosas, que retornam à atmosfera, sobretudo pelos processos de desnitrificação e volatilização (Siqueira et al.,1994; Straliotto et al., 2002).

Nesse contexto, o manejo adequado da adubação nitrogenada representa uma das principais dificuldades da cultura do feijoeiro, visto que a aplicação de doses excessivas de N, além de aumentar o custo econômico, pode promover sérios riscos ao ambiente, e a sua utilização em quantidade insuficiente pode limitar o seu potencial produtivo, mesmo que outros fatores de produção sejam otimizados (Santos et al., 2003). 
Por outro lado, estudos têm demonstrado que é possível que essa cultura se beneficie, em condições de campo, do processo de fixação biológica de $\mathrm{N}_{2}$, podendo alcançar produtividade acima de $2.500 \mathrm{~kg} \mathrm{ha}^{-1}$ (Hungria et al., 2000). Deve-se, contudo, considerar que o sucesso de inoculação do feijoeiro com estirpes de rizóbio com alta eficiência está associado à habilidade competitiva de tais estirpes e adaptação às condições ambientais (Mercante et al., 1999; Straliotto et al., 2002). Sob condições ambientais adequadas, o $\mathrm{N}_{2}$ atmosférico fixado pela simbiose pode atender à maior parte das necessidades do feijoeiro (Hungria et al., 1985). Entretanto, os fatores da acidez do solo, $\mathrm{pH}$ baixo e concentrações elevadas de $\mathrm{Al}$ tóxico, frequentemente, limitam todas as etapas do processo de infecção das raízes, formação de nódulos e assimilação do N pela planta (Dernadin, 1991; Martinez-Romero et al., 1991; Mercante, 1993).

O presente estudo teve como objetivo avaliar a resposta do feijoeiro à inoculação com rizóbio e ao parcelamento de fertilizante nitrogenado, em termos de nodulação das plantas e produtividade de grãos da cultura, bem como a viabilidade econômica da aplicação de fertilizante nitrogenado e, ou, inoculação com rizóbio em feijoeiro.

\section{MATERIAL E MÉTODOS}

O experimento foi realizado num Latossolo Vermelho distroférrico, na área experimental da Fazenda-Escola da UNIDERP- Universidade para o Desenvolvimento do Estado e da Região do Pantanal, município de Dourados-MS (22 ${ }^{\circ} 16^{\prime}$ ' S e $54^{\circ} 49^{\prime}$ W). A análise química do solo mostrou os seguintes valores: pH em água de 5,9; MO de 32,7 $\mathrm{g} \mathrm{kg}^{-1} ; \mathrm{P}$ (Mehlich-1) de $12,3 \mathrm{mg} \mathrm{dm}{ }^{-3} ; \mathrm{Al}^{3+}, \mathrm{Ca}^{2+}, \mathrm{Mg}^{2+}, \mathrm{H}+\mathrm{Al}$ e K de 0,0; 7,$1 ; 2,7 ; 4,7$ e $0,82 \mathrm{cmol}_{\mathrm{c}} \mathrm{dm}^{-3}$, respectivamente; e $\mathrm{V}=69 \%$. Durante o cultivo, a precipitação pluvial foi de $231,2 \mathrm{~mm}$, a temperatura média foi de $20^{\circ} \mathrm{C}$, com máxima de $34,4^{\circ} \mathrm{C}$ e mínima de $2,8^{\circ} \mathrm{C}$, e a umidade relativa média do ar foi de $72 \%$, tendo variado entre 22 e $100 \%$. Como a precipitação pluvial no período de condução do experimento foi baixa, foi realizada irrigação, via aspersão, quando necessária.

A semeadura do feijoeiro, cultivar Pérola, foi realizada manualmente em 4/04/2007, no sistema de plantio convencional sobre área em pousio (várias espécies de plantas espontâneas, com predomínio de Brachiaria decumbens), utilizando-se 15 sementes por metro linear, com espaçamento entre linhas de $0,45 \mathrm{~m}$. A adubação básica constou da aplicação de $320 \mathrm{~kg} \mathrm{ha}^{-1}$ da formulação 0-20-20 (N-P-K), no sulco de plantio, com base na análise do solo. O controle de plantas daninhas foi feito por capina manual, e os tratamentos fitossanitários, mediante aplicação de produtos recomendados para a cultura. O cultivo ocorreu durante o período de outono/inverno.
O delineamento experimental utilizado foi o de blocos casualizados, com seis repetições. Os tratamentos consistiram de: (1) testemunha sem inoculação e sem aplicação de N; (2) inoculação com rizóbio e sem aplicação de N; (3) inoculação de rizóbio e aplicação de $20 \mathrm{~kg} \mathrm{ha}^{-1}$ de N, na semeadura; (4) aplicação de $20 \mathrm{~kg} \mathrm{ha}^{-1}$ de $\mathrm{N}$ na semeadura e sem inoculação com rizóbio; (5) aplicação de $40 \mathrm{~kg} \mathrm{ha}^{-1} \mathrm{de}$ $\mathrm{N}\left(20 \mathrm{~kg} \mathrm{ha}^{-1}\right.$ na semeadura e $20 \mathrm{~kg} \mathrm{ha}^{-1}$ aos 20 dias após a emergência das plantas - DAE) e sem inoculação; (6) aplicação de $80 \mathrm{~kg} \mathrm{ha}^{-1}$ de N $\left(20 \mathrm{~kg} \mathrm{ha}^{-1}\right.$ na semeadura, $30 \mathrm{~kg} \mathrm{ha}^{-1}$ aos 20 DAE e $30 \mathrm{~kg} \mathrm{ha}^{-1}$ aos 40 DAE) e sem inoculação com rizóbio; e (7) aplicação de $160 \mathrm{~kg} \mathrm{ha}^{-1}$ de $\mathrm{N}\left(20 \mathrm{~kg} \mathrm{ha}^{-1}\right.$ no plantio, $70 \mathrm{~kg} \mathrm{ha}^{-1}$ aos 20 DAE e $70 \mathrm{~kg} \mathrm{ha}^{-1}$ aos $\left.40 \mathrm{DAE}\right)$ e sem inoculação. Cada parcela foi composta por seis linhas de $4 \mathrm{~m}$ de comprimento. Nas adubações de cobertura, utilizouse ureia, aplicada manualmente e a lanço.

As sementes foram inoculadas com a estirpe de $R$. tropici CIAT 899 (SEMIA 4077), recomendada comercialmente para o feijoeiro no Brasil, sendo o produto preparado a uma densidade de $10^{9}$ células $\mathrm{g}^{-1}$ de turfa. $\mathrm{O}$ inoculante foi adicionado às sementes na proporção de $500 \mathrm{~g}$ para $50 \mathrm{~kg}$ de sementes, acrescentando-se $300 \mathrm{~mL}$ de solução açucarada a $10 \%$ $(\mathrm{p}: \mathrm{v})$, visando à melhoria de sua aderência às sementes.

Foram realizadas as seguintes avaliações: número de nódulos, matéria seca de nódulos (secagem em estufa a $50^{\circ} \mathrm{C}$, até peso constante), matéria seca da parte aérea (secagem em estufa a $50^{\circ} \mathrm{C}$, até peso constante), teor de $\mathrm{N}$ total da parte aérea, peso de 100 sementes, teor de $\mathrm{N}$ nos grãos, proteína bruta nos grãos e produtividade de grãos (corrigida para $13 \%$ de umidade). As avaliações de nodulação, matéria seca e teor de $\mathrm{N}$ total da parte aérea foram realizadas em plantas coletadas aos $37 \mathrm{DAE}$ (florescimento pleno), numa linha entre duas bordaduras. Para avaliação da produtividade de grãos, foram consideradas duas linhas entre bordaduras, correspondendo a uma área útil de $3,6 \mathrm{~m}^{2}$ por parcela. O teor de $\mathrm{N}$, tanto da parte aérea quanto dos grãos, foi determinado pelo método de Kjeldahl (semimicro), segundo Malavolta et al. (1997). Para determinação da proteína bruta nos grãos, utilizou-se o fator de correção de 6,25 para os teores de N nos grãos.

Para realização da análise econômica, foram considerados os custos da ureia e sua aplicação (a lanço), além do custo do inoculante; os demais custos, como o plantio e tratos culturais, não foram considerados, por serem os mesmos para todos os tratamentos. O preço da tonelada de ureia foi de $\mathrm{R} \$ 1.000,00$ (preço comercial médio entre março e maio de 2007); logo, o custo por quilo de $\mathrm{N}$ foi de $\mathrm{R} \$ 2,22$ (CONAB, 2007). O custo estimado da aplicação de ureia a lanço foi de $R \$ 17,30$ por hectare, e o custo da dose de inoculante, de $\mathrm{R} \$ 5,00$ por ha. O preço médio da saca de $60 \mathrm{~kg}$ de feijão carioca foi de $\mathrm{R} \$ 50,00$. Comparou-se o incremento de produtividade, o custo de produção, o acréscimo da 
receita bruta e o acréscimo da receita líquida, em relação à testemunha sem inoculação e sem adubação nitrogenada. Entende-se por incremento de produtividade a diferença entre a produtividade de grãos correspondente a cada um dos tratamentos em relação ao tratamento sem inoculação e sem adubação (controle). O acréscimo de receita bruta foi determinado pelo acréscimo de produtividade multiplicado pelo preço de venda do feijão carioca. $\mathrm{O}$ acréscimo de receita líquida foi obtido pela diferença entre o acréscimo de receita bruta e os custos da adubação nitrogenada, sua aplicação e, ou, inoculação.

Os resultados foram submetidos à análise de variância, sendo as médias dos tratamentos comparadas pelo teste de Tukey a $5 \%$, por meio do programa de estatística ASSISTAT (Silva \& Azevedo, 2002). Realizou-se também regressão polinomial, para avaliação da resposta de cada variável em relação às doses de $\mathrm{N}$ aplicadas; neste caso, foram excluídos os tratamentos com uso do inoculante. Essa análise foi realizada por meio do programa estatístico SISVAR (Ferreira, 2000).

\section{RESULTADOS E DISCUSSÃO}

Verificou-se que não houve diferença significativa $(p<0,05)$ entre os tratamentos para nodulação (número e matéria seca de nódulos) de feijoeiros (Quadro 1). A nodulação das plantas de feijoeiro que não foram inoculadas nem receberam aplicação de $\mathrm{N}$ mineral (testemunha absoluta) foi similar à dos demais tratamentos, evidenciando a existência de elevada e eficiente população nativa de rizóbios simbiontes do feijoeiro. Contudo, verificou-se tendência de diminuição da nodulação (número e matéria seca de nódulos) conforme o aumento da dose de $\mathrm{N}$ aplicada. Essa tendência se confirma por meio da análise de regressão, que demonstrou efeito linear significativo para redução do número de nódulos (Figura 1a) e matéria seca de nódulos (Figura 1b) com o aumento das doses de N. Do mesmo modo, Hungria et al. (2003) não observaram diferenças significativas no número de nódulos de feijoeiros inoculados com rizóbios eficientes e plantas supridas com $\mathrm{N}$ mineral $\left(30 \mathrm{~kg} \mathrm{ha}^{-1}\right.$ no plantio $+30 \mathrm{~kg} \mathrm{ha}^{-1}$ em cobertura) em relação à população nativa do solo na região Sul do País. Em solos do Cerrado, Peres et al. (1994) observaram incrementos significativos na nodulação de feijoeiros em três ensaios, quando utilizada a inoculação com estirpes comerciais de rizóbios, para a cultivar Carioca, e diminuição da nodulação com a aplicação de $\mathrm{N}$ mineral. Em outro estudo realizado em solo do Mato Grosso do Sul, Mercante et al. (2006) observaram nodulação superior nas plantas inoculadas com estirpes de rizóbios eficientes (CIAT 899 e PRF 81), quando comparadas com as plantas noduladas com os rizóbios nativos e com os tratamentos com adubação mineral nitrogenada, para todas as quatro cultivares avaliadas.

Quanto à produção de matéria seca da parte aérea, não foram detectadas diferenças significativas $(p<0,05)$ entre os diferentes tratamentos avaliados (Quadro 1) e também não houve ajuste significativo de um modelo de regressão em relação às doses de $\mathrm{N}$ aplicadas. Contudo, os valores mais elevados foram verificados no tratamento com aplicação de $40 \mathrm{~kg} \mathrm{ha}^{-1}$ de $\mathrm{N}$ e sem uso de inoculante $(20,20,0)$. Em geral, as respostas do feijoeiro à adubação nitrogenada têm sido bastante variáveis quanto à produção de matéria seca da parte aérea, sendo observados efeitos positivos (Carvalho et al., 2001) ou sem efeitos significativos (Soratto et al., 2006; Farinelli et al., 2006), como no presente trabalho, embora promovendo aumento linear com as doses utilizadas.

Quadro 1. Número de nódulos, matéria seca de nódulos e da parte aérea, teor de $\mathrm{N}$ total da parte aérea e de grãos, massa de cem sementes e produtividade de grãos de feijoeiro, cv. Pérola, supridos ou não com $\mathrm{N}$ e, ou, inoculados com rizóbio

\begin{tabular}{|c|c|c|c|c|c|c|c|}
\hline Tratamento & $\begin{array}{c}\text { Número } \\
\text { de } \\
\text { nódulos }\end{array}$ & $\begin{array}{c}\text { Matéria } \\
\text { seca } \\
\text { de nódulos }\end{array}$ & $\begin{array}{c}\text { Matéria seca } \\
\text { da parte } \\
\text { aérea }\end{array}$ & $\begin{array}{c}\text { Teor de } \mathrm{N} \\
\text { na parte } \\
\text { aérea }\end{array}$ & $\begin{array}{l}\text { Massa de } \\
\text { cem } \\
\text { sementes }\end{array}$ & $\begin{array}{l}\text { Teor de } \\
\text { N nos } \\
\text { grãos }\end{array}$ & $\begin{array}{c}\text { Produtividade } \\
\text { de grãos }\end{array}$ \\
\hline & $\mathrm{n}^{\mathrm{o}} \mathrm{pl}^{-1}$ & $\mathrm{mg} \mathrm{pl}^{-1}$ & $\mathrm{~g} \mathrm{pl}^{-1}$ & $\mathrm{~g} \mathrm{~kg}^{-1}$ & $\mathrm{~g}$ & $\mathrm{~g} \mathrm{~kg}^{-1}$ & $\mathrm{~kg} \mathrm{ha} \mathrm{h}^{-1}$ \\
\hline 1. Sem N & $14,2 \mathrm{a}$ & 41,9 a & $3,9 \mathrm{a}$ & $36,7 \mathrm{a}$ & $28,8 \mathrm{a}$ & 33,4 a & $2.967 \mathrm{c}$ \\
\hline 2. Inoculado & $12,6 \mathrm{a}$ & $42,3 \mathrm{a}$ & $3,8 \mathrm{a}$ & $32,8 \mathrm{a}$ & $30,1 \mathrm{a}$ & $32,7 \mathrm{a}$ & $3.131 \mathrm{bc}$ \\
\hline 3. Inoculado $+20 \mathrm{~kg} \mathrm{ha}^{-1}$ & $13,5 \mathrm{a}$ & $44,3 \mathrm{a}$ & $3,9 \mathrm{a}$ & $33,4 \mathrm{a}$ & $29,3 \mathrm{a}$ & $32,5 \mathrm{a}$ & $3.339 \mathrm{abc}$ \\
\hline 4. $20 \mathrm{~kg} \mathrm{ha}^{-1}$ & $11,3 \mathrm{a}$ & $33,1 \mathrm{a}$ & $4,1 \mathrm{a}$ & 33,5 a & 29,6 a & $33,2 \mathrm{a}$ & $3.261 \mathrm{abc}$ \\
\hline 5. $40 \mathrm{~kg} \mathrm{ha}^{-1}$ de $\mathrm{N}(20+20)$ & $11,9 \mathrm{a}$ & 33,9 a & $4,9 \mathrm{a}$ & 35,0 a & $28,8 \mathrm{a}$ & $32,9 \mathrm{a}$ & $3.666 \mathrm{ab}$ \\
\hline $6.80(20+30+30)$ & $7,4 \mathrm{a}$ & $20,1 \mathrm{a}$ & $4,1 \mathrm{a}$ & $35,1 \mathrm{a}$ & $30,7 \mathrm{a}$ & $33,2 \mathrm{a}$ & $3.623 \mathrm{ab}$ \\
\hline 7. $160(20+70+70)$ & $2,6 \mathrm{a}$ & $4,4 \mathrm{a}$ & $4,6 \mathrm{a}$ & $36,6 \mathrm{a}$ & $30,6 \mathrm{a}$ & $35,1 \mathrm{a}$ & $3.762 \mathrm{a}$ \\
\hline CV (\%) & 75,9 & 81,8 & 22,9 & 6,4 & 4,0 & 4,6 & 10,1 \\
\hline
\end{tabular}

Médias seguidas de mesmas letras, na coluna, não diferem entre si pelo teste de Tukey a $5 \%$. 
Não houve diferença significativa $(p<0,05)$ entre os tratamentos para o teor de $\mathrm{N}$ na parte aérea (Quadro 1). Entretanto, deve-se salientar que para todos os tratamentos esses teores ficaram acima do nível crítico de $\mathrm{N}$ para a cultura, de $30 \mathrm{~g} \mathrm{~kg}^{-1}$, segundo Ambrosano et al. (1997), ou 30-50 $\mathrm{g} \mathrm{kg}^{-1}$, conforme Malavolta et al. (1997). Esses níveis de suficiência de $\mathrm{N}$ na parte aérea de feijoeiros têm sido verificados em solos mais férteis ou com populações de rizóbio nativo abundantes e de elevada eficiência simbiótica (Almeida et al., 2000; Soratto et al., 2006; Farinelli et al., 2006). Por outro lado, em solos mais pobres em $\mathrm{N}$ e com baixas populações de rizóbios com eficiência simbiótica elevada, o efeito da aplicação de fertilizantes nitrogenados tem proporcionado teores foliares superiores aos verificados nas plantas-testemunha, sem adubação nitrogenada (Carvalho et al., 2001; Mercante et al., 2006).

$\mathrm{Na}$ análise da massa de cem sementes de feijão (Quadro 1) não foi detectada diferença significativa $(p<0,05)$ entre os tratamentos na comparação das médias, com valores entre 28,8 e 30,7 g. Esses valores estão acima de $27 \mathrm{~g}$, considerado característico da cultivar Pérola (Cultivar..., 2007). Na análise de regressão foi detectado efeito linear de aumento da massa de cem grãos, em relação às doses de $\mathrm{N}$ aplicadas (Figura 1c), conforme observado também nos estudos de Soratto et al. (2006) e Farinelli et al. (2006).
Do mesmo modo que nas variáveis já mencionadas, para o teor de $\mathrm{N}$ nos grãos (Quadro 1), não houve diferença significativa entre os tratamentos. Também não houve resposta significativa na análise de regressão em relação às doses de $\mathrm{N}$ aplicadas. Em outro estudo com feijoeiro, Farinelli et al. (2006) observaram teores de proteína bruta próximos aos verificados no presente trabalho, porém com aumento linear em relação às doses de $\mathrm{N}$ aplicadas. Soratto et al. (2005) observaram que a aplicação de $\mathrm{N}$ em cobertura, nos estádios $\mathrm{V}_{4}$ e início do $\mathrm{R}_{7}$, proporcionou aumento no teor de proteína nos grãos do feijoeiro.

A produtividade de grãos variou entre $2.967 \mathrm{e}$ $3.762 \mathrm{~kg} \mathrm{ha}^{-1}$ (Quadro 1). Esses valores são bastante superiores à média verificada em Mato Grosso do Sul, nas safras de 2004/05 e 2005/06, que foram de 1.100 e $945 \mathrm{~kg} \mathrm{ha}^{-1}$, respectivamente (CONAB, 2008). A produtividade mais elevada $\left(3.762 \mathrm{~kg} \mathrm{ha}^{-1}\right)$, correspondente ao tratamento com aplicação de $160 \mathrm{~kg} \mathrm{ha}^{-1}$, esteve próxima ao potencial de produção da cultivar, que é de aproximadamente $4.000 \mathrm{~kg} \mathrm{ha}^{-1}$ (Cultivar..., 2007). Contudo, o valor de produtividade de grãos com aplicação de $160 \mathrm{~kg} \mathrm{ha}^{-1}$ não diferiu significativamente dos tratamentos com aplicação das demais doses de $\mathrm{N}$, sendo superior apenas aos tratamentos sem aplicação de $\mathrm{N}$ (com e sem inoculação com rizóbio). Não foram detectadas diferenças significativas entre os tratamentos que receberam
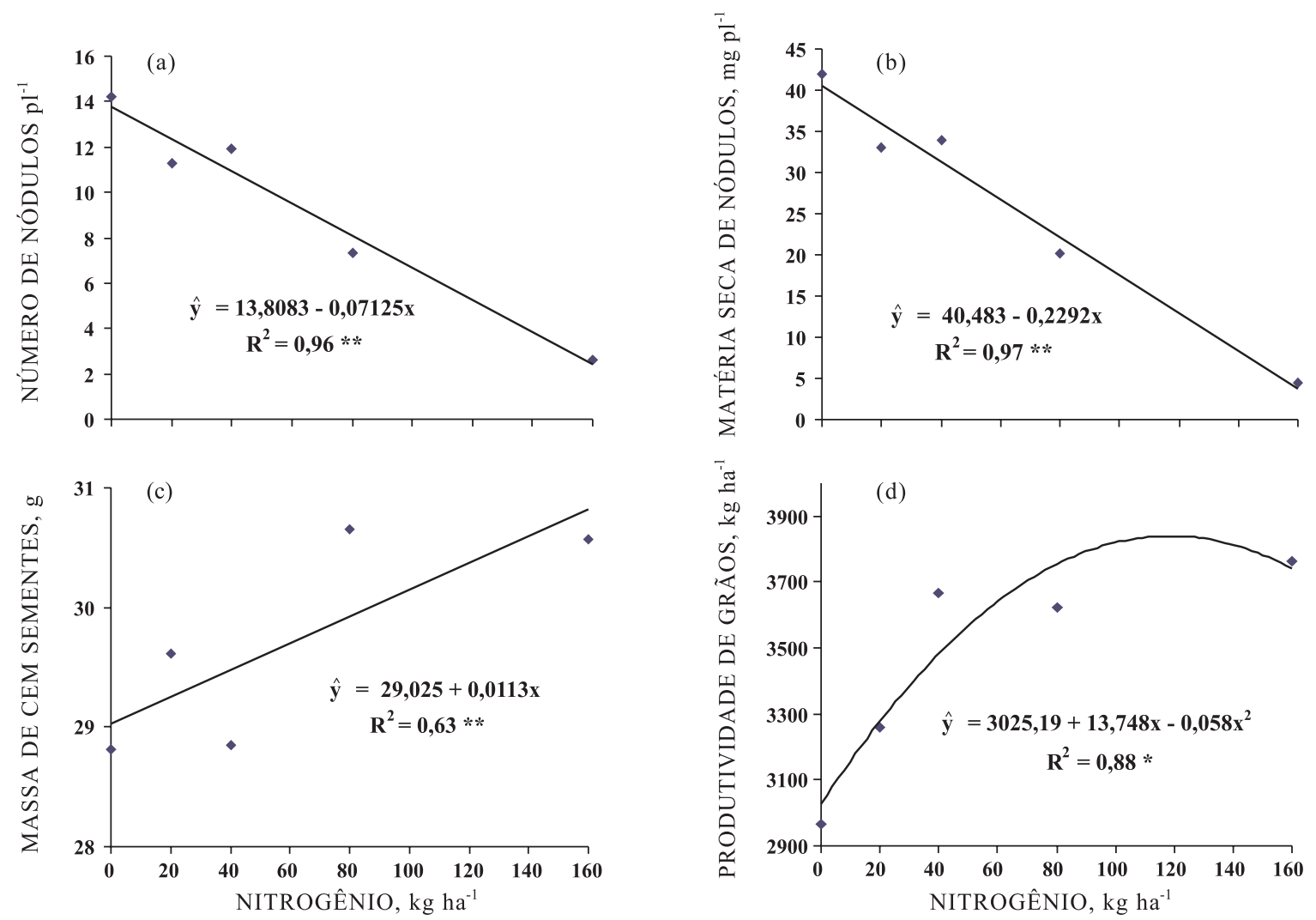

Figura 1. Número de nódulos (a), matéria seca de nódulos (b), massa de cem sementes (c) e produtividade de grãos de feijoeiro (d) em função da quantidade de nitrogênio fornecida, cv. Pérola. ** $\mathrm{e}^{*}$ : significativo a 1 e $5 \%$, respectivamente. 
adubação nitrogenada. A aplicação de $20 \mathrm{~kg} \mathrm{ha}^{-1} \mathrm{de}$ $\mathrm{N}$ não promoveu incrementos significativos em comparação com os tratamentos que não receberam adubação nitrogenada (com e sem inoculação). As doses acima de $20 \mathrm{~kg} \mathrm{ha}^{-1}$ de $\mathrm{N}$ proporcionaram acréscimos significativos na produtividade de grãos, quando comparadas às dos tratamentos sem adubação nitrogenada. Houve resposta quadrática para as produtividades obtidas, em que o valor máximo seria alcançado com uma dose de $116 \mathrm{~kg} \mathrm{ha}^{-1}$ de $\mathrm{N}$ (Figura 1d).

De modo geral, as respostas da produção de grãos à adubação nitogenada têm sido bastante variáveis em outras condições e localidades do Brasil. Nos estudos de Soratto et al. (2006), realizados na região leste de Mato Grosso do Sul, também foi verificada resposta quadrática para a produtividade de grãos, com a dose estimada para a máxima produtividade superior a $140 \mathrm{~kg} \mathrm{ha}^{-1}$ de N. Em outro ensaio desses estudos, os autores obtiveram resposta linear na produtividade de grãos. No Estado de São Paulo, Farinelli et al. (2006) avaliaram a aplicação de diversas doses de $\mathrm{N}$ em cobertura na cultura do feijoeiro, manejado em plantio direto e plantio convencional, e também verificaram ajuste quadrático para a produtividade no primeiro ano, sendo a produtividade mais elevada alcançada com a aplicação de $78 \mathrm{~kg} \mathrm{ha}^{-1}$ de N. No segundo ano, obtiveram resposta linear no sistema plantio direto e quadrática para o sistema convencional, sendo a produtividade máxima alcançada com a aplicação de $185 \mathrm{~kg} \mathrm{ha}^{-1}$ de N. Em outro ensaio realizado em Mato Grosso do Sul, a produtividade máxima de grãos do feijoeiro foi alcançada com aplicação de $140 \mathrm{~kg} \mathrm{ha}^{-1}$ de N, para três das quatro cultivares avaliadas (Mercante et al., 2006). Essa variabilidade nas respostas de produtividade às doses de $\mathrm{N}$, nos diferentes locais, tem sido verificada especialmente em função dos níveis de fertilidade do solo e outras técnicas empregadas nos sistemas produtivos, destacando-se o uso de sistemas de irrigação.

Pode-se observar que todos os tratamentos correspondentes à inoculação e, ou, aplicação de adubo nitrogenado apresentaram acréscimos de produtividade, de receita bruta e receita líquida, em relação ao tratamento testemunha (sem inoculação e sem adubação nitrogenada) (Quadro 2). O maior incremento de produtividade ocorreu com a aplicação de $160 \mathrm{~kg} \mathrm{ha}^{-1}$ de $\mathrm{N}$, chegando a $795 \mathrm{~kg} \mathrm{ha}^{-1}$, seguido pela aplicação de $40 \mathrm{~kg} \mathrm{ha}^{-1}$ de N, com $699 \mathrm{~kg} \mathrm{ha}^{-1}$; o menor incremento foi verificado no tratamento com inoculação e sem adubação nitrogenada, com média de $165 \mathrm{~kg} \mathrm{ha}^{-1}$.

Quanto ao acréscimo de receita líquida (Quadro 2), observa-se que a aplicação de $40 \mathrm{~kg} \mathrm{ha}^{-1}$ de $\mathrm{N}$ proporcionou o maior acréscimo, atingindo $\mathrm{R} \$ 477 \mathrm{ha}^{-1}$, seguido pelo tratamento correspondente à aplicação de $80 \mathrm{~kg} \mathrm{ha}^{-1}$ de $\mathrm{N}$, com acréscimo de $\mathrm{R} \$ 335 \mathrm{ha}^{-1}$, e pela aplicação de $160 \mathrm{~kg} \mathrm{ha}^{-1} \mathrm{de} \mathrm{N}$, com acréscimo de $\mathrm{R} \$ 273 \mathrm{ha}^{-1}$. Deve-se destacar que, apesar do maior incremento de produtividade com a aplicação de $160 \mathrm{~kg} \mathrm{ha}^{-1}$ de $\mathrm{N}$, chegando aos $795 \mathrm{~kg} \mathrm{ha}^{-1}$, este tratamento apresentou acréscimo de receita líquida de apenas $\mathrm{R} \$ 273 \mathrm{ha}^{-1}$, sendo comparável ao do tratamento com uso da inoculação e aplicação de $20 \mathrm{~kg} \mathrm{ha}^{-1}$ de $\mathrm{N}$ no plantio, que atingiu $\mathrm{R} \$ 261 \mathrm{ha}^{-1}$, embora tenha apresentado incremento de produtividade de apenas $373 \mathrm{~kg} \mathrm{ha}^{-1}$. Isso se deve, principalmente, ao alto custo da adubação nitrogenada empregada no sistema com a aplicação de $160 \mathrm{~kg} \mathrm{ha}^{-1}$ de N. Deve-se observar, ainda, que o uso da inoculação de rizóbio, acrescida da adubação com $20 \mathrm{~kg} \mathrm{ha}^{-1} \mathrm{de}$ $\mathrm{N}$ no plantio, apresentou incrementos de produtividade e de receita líquida superiores aos do tratamento com aplicação exclusiva de $20 \mathrm{~kg} \mathrm{ha}^{-1}$ de $\mathrm{N}$ no plantio e ao tratamento em que houve apenas a inoculação. Binotti

Quadro 2. Acréscimo de produtividade e acréscimo de receita bruta e líquida devido à adubação nitrogenada e custo total da adubação nitrogenada

\begin{tabular}{|c|c|c|c|c|}
\hline Tratamento & $\begin{array}{c}\text { Acréscimo de } \\
\text { produtividade }^{(1)}\end{array}$ & $\begin{array}{l}\text { Acréscimo de } \\
\text { receita bruta }^{(2)}\end{array}$ & $\begin{array}{l}\text { Custo total } \\
\text { adubação }^{(3)}\end{array}$ & $\begin{array}{l}\text { Acréscimo de } \\
\text { receita líquida }\end{array}$ \\
\hline & $\mathrm{kg} \mathrm{ha}^{-1}$ & $\mathrm{R} \$ \mathrm{ha}^{-1}$ & $\mathrm{R} \$ \mathrm{ha}^{-1}$ & $\mathrm{R} \$ \mathrm{ha}^{-1}$ \\
\hline 1. Sem inoculado e sem $\mathrm{N}$ & 0 & 0 & 0 & 0 \\
\hline 2. Inoculado & 165 & 137 & 5 & 132 \\
\hline 3. Inoculado $+20 \mathrm{~kg} \mathrm{ha}^{-1}$ & 373 & 311 & 49 & 262 \\
\hline 4. $20 \mathrm{~kg} \mathrm{ha}^{-1}$ & 294 & 245 & 44 & 201 \\
\hline 5. $40 \mathrm{~kg} \mathrm{ha}^{-1}$ de $\mathrm{N}(20+20)$ & 699 & 583 & 106 & 477 \\
\hline $6.80(20+30+30)$ & 656 & 547 & 212 & 335 \\
\hline 7. $160(20+70+70)$ & 795 & 663 & 390 & 273 \\
\hline
\end{tabular}

${ }^{(1)}$ Acréscimo de produtividade em relação a testemunha sem inoculação e sem adubação nitrogenada. ${ }^{(2)}$ Considerando $\mathrm{R} \$ 50,00$ a saca de $60 \mathrm{~kg}$ de feijão carioca. ${ }^{(3)}$ Tratamento 2: somente o custo do inoculante; tratamento $3:$ custo do inoculante mais $20 \mathrm{~kg} \mathrm{ha}^{-1}$ de $\mathrm{N}$ no plantio; tratamento 4: custo de $20 \mathrm{~kg} \mathrm{ha}^{-1}$ de $\mathrm{N}$ no plantio; tratamento 5: custo de $20 \mathrm{~kg}^{-1}$ de $\mathrm{N}$ no plantio + $20 \mathrm{~kg} \mathrm{ha}^{-1}$ de $\mathrm{N}$ aos $20 \mathrm{DAE}$ (a lanço); tratamento 6: custo de $20 \mathrm{~kg} \mathrm{ha}^{-1}$ de $\mathrm{N}$ no plantio $+30 \mathrm{~kg} \mathrm{ha}^{-1}$ de $\mathrm{N}$ aos $20 \mathrm{DAE}(\mathrm{a}$ lanço) + $30 \mathrm{~kg} \mathrm{ha}^{-1}$ de $\mathrm{N}$ aos $40 \mathrm{DAE}$ (a lanço); tratamento 7: custo de $20 \mathrm{~kg} \mathrm{ha}^{-1}$ de $\mathrm{N}$ no plantio $+70 \mathrm{~kg} \mathrm{ha}^{-1}$ de $\mathrm{N}$ aos $20 \mathrm{DAE}$ (a lanço) + $70 \mathrm{~kg} \mathrm{ha}^{-1}$ de $\mathrm{N}$ aos 40 DAE (a lanço). 
et al. (2007), testando diferentes fontes e doses de N, aplicadas em épocas diferenciadas, observaram os maiores incrementos de produtividade $\left(1.158 \mathrm{~kg} \mathrm{ha}^{-1}\right)$ no tratamento com aplicação de $200 \mathrm{~kg} \mathrm{ha}^{-1} \mathrm{de} \mathrm{N}$ na forma de sulfato de amônio, porém a maior margem bruta (já descontado o custo da adubação nitrogenada) foi de $\mathrm{R} \$ 483 \mathrm{ha}^{-1}$ para a mistura de $1 / 2$ de $\mathrm{N}$ da ureia $+1 / 2$ de $\mathrm{N}$ do sulfato de amônio, na dose de $150 \mathrm{~kg} \mathrm{ha}-1$ de N, $100 \%$ na semeadura. De modo geral, esses autores observaram que o uso de ureia como fonte de $\mathrm{N}$ proporcionou maior margem bruta em quase todos os tratamentos. A aplicação de $50 \mathrm{~kg} \mathrm{ha}^{-1}$ de $\mathrm{N}$ (1/3 semeadura $+1 / 3$ cobertura aos 23 DAE) proporcionou uma margem de ganho bruto de $\mathrm{R} \$ 430 \mathrm{ha}^{-1}$, e a aplicação de $150 \mathrm{~kg} \mathrm{ha}^{-1}$ de N (1/3 semeadura + 1/3 cobertura aos 23 DAE) resultou numa margem de ganho bruto de $\mathrm{R} \$ 250 \mathrm{ha}^{-1}$ (Binotti et al., 2007). Esses incrementos foram similares aos obtidos no presente estudo, indicando que adubações entre 40 e $50 \mathrm{~kg} \mathrm{ha}^{-1}$ de $\mathrm{N}$ podem representar maior rentabilidade para os agricultores.

\section{CONCLUSÕES}

1. A adubação com $20 \mathrm{~kg} \mathrm{ha}^{-1}$ de $\mathrm{N}$, acrescida de inoculante com a estirpe de $R$. tropici CIAT 899 possibilitou a obtenção de rendimento de grãos na cultura de feijoeiro equivalente à aplicação de até 160 $\mathrm{kg} \mathrm{ha}{ }^{-1}$ de N.

2. A inoculação de rizóbio, acrescida da adubação com $20 \mathrm{~kg} \mathrm{ha}^{-1}$ de $\mathrm{N}$ no plantio, propiciou acréscimo de receita líquida semelhante à aplicação de $160 \mathrm{~kg} \mathrm{ha}^{-1}$ de $\mathrm{N}$ e superior ao tratamento com a adubação de $20 \mathrm{~kg} \mathrm{ha}^{-1}$ de N, sem o uso do inoculante, evidenciando a sua importância para obtenção de maior rentabilidade na cultura do feijoeiro.

\section{LITERATURA CITADA}

ALMEIDA, C.; CARVALHO, M.A.C.; ARF, O.; SÁ, M.E. \& BUZETTI, S. Ureia em cobertura e via foliar em feijoeiro. Sci. Agric., 57:293-298, 2000.

AMBROSANO, E.J.; TANAKA, R.T.; MASCARENHAS, H.A.A.; RAIJ, B.van; QUAGGIO, J.A. \& CANTARELLA, H. Leguminosas e oleaginosas. In: RAIJ, B.van; CANTARELA, H.; QUAGGIO, J.A. \& FURLANI, A.M.C., eds. Recomendações de adubação e calagem para o Estado de São Paulo. Campinas, Instituto Agronômico de Campinas, 1997. p.187-203. (Boletim Técnico, 100)

BINOTTI, F.F.S.; ARF, O.; SÁ, M.E.; BUZETTI, S.; ALVAREZ, A.C.C. \& KAMIMURA, K.M. Manejo do nitrogênio em feijoeiro de inverno no sistema plantio direto - II Análise econômica. In: CONGRESSO BRASILEIRO DE CIÊNCIA DO SOLO, 31., Gramado, 2007. Conquistas e desafios da ciência do solo brasileiro. Gramado, Sociedade Brasileira de Ciência do Solo; Universidade Federal do Rio Grande do Sul, 2007. CD-ROM.
CABALLERO, S.V.; LIBARDI, P.L.; REICHARDT, K.; MATSUI, E. \& VICTORIA, R.L. Utilização de fertilizante nitrogenado aplicado a uma cultura de feijão. Pesq. Agropec. Bras., 20:1031-1040, 1985.

CARVALHO, M.A.C.; ARF, O.; SÁ, M.E.; BUZETTI, S.; SANTOS, N.C.B. \& BASSAN, D.A.Z. Produtividade e qualidade de sementes de feijoeiro (Phaseolus vulgaris L.) sob influência de parcelamentos e fontes de nitrogênio. R. Bras. Ci. Solo, 25:617-624, 2001.

CONAB. Indicadores agropecuários. Tabelas de preços de agrotóxicos. Disponível em: <http://www.conab.gov.br/ conabweb/index.php?PAG=213>. Acesso em: 10 out. de 2007.

CONAB. Feijão total $\left(1^{\mathrm{a}}, 2^{\mathrm{a}}\right.$ e $3^{\mathrm{a}}$ safras). Disponível em: $<\mathrm{http} / /$ /www.conab.gov.br/conabweb/download/safra/ FeijaoTotalSerieHist.xls>. Acesso em: 21 fev. de 2008.

CULTIVAR de feijão: Pérola. Disponível em: <http:// guapore.cnpaf.embrapa.br/feijao/perola.htm>. Acesso em: 17 out. de 2007.

DERNADIN, N.D. Seleção de estirpes de Rhizobium leguminosarum bv. phaseoli tolerantes a fatores de acidez e resistentes a antibióticos. Piracicaba, Escola Superior de Agricultura Luiz de Queiroz, 1991. 89p. (Tese de Mestrado).

FARINELLI, R.; LEMOS, L.B.; PENARIOL, F.G.; EGÉA, M.M. \& GASPAROTO, M.G. Adubação nitrogenada de cobertura no feijoeiro, em plantio direto e convencional. Pesq. Agropec. Bras., 41:307-312, 2006.

FERREIRA, D.F. Análises estatísticas por meio do SISVAR (Sistema para Análise de Variância) para Windows versão 4.0. In: REUNIÃO ANUAL DA REGIÃO BRASILEIRA DA SOCIEDADE INTERNACIONAL DE BIOMETRIA, 45., São Carlos, 2000. Anais. São Carlos, Universidade Federal de São Carlos, 2000. p.255-258.

HUNGRIA, M.; ANDRADE, D.S.; CHUEIRE, L.M.O.; PROBANZA, A.; GUTIERREZ-MAÑERO, F.J. \& MEGIAS, M. Isolation and characterization of new efficient and competitive bean (Phaseolus vulgaris L.) rhizobia from Brazil. Soil Biol. Biochem., 32:1515-1528, 2000.

HUNGRIA, M.; NEVES, M.C.P. \& VICTORIA, R.L. Assimilação do nitrogênio pelo feijoeiro; II. Absorção e translocação do $\mathrm{N}$ mineral e do $\mathrm{N}_{2}$ fixado. R. Bras. Ci. Solo, 9:202-209, 1985.

HUNGRIA, M.; CAMPO, R.J. \& MENDES, I. Benefits of inoculation of the common bean (Phaseolus vulgaris) crop with efficient and competitive Rhizobium tropici strains. Biol. Fert. Soils, 39:88-93, 2003.

HUNGRIA, M.; VARGAS, M.A.T. \& ARAUJO, R.S. Fixação biológica de nitrogênio em feijoeiro. In: VARGAS, M.A.T. \& HUNGRIA, M., eds. Biologia dos solos dos cerrados. Planaltina, Embrapa-CPAC, 1997. p.189-294.

MALAVOLTA, E.; VITTI, G.C. \& OLIVEIRA, S.A. Avaliação do estado nutricional das plantas: Princípios e aplicações. 2.ed. Piracicaba, POTAFOS, 1997. 319p. 
MARTÍNEZ-ROMERO, E.; SEGOVIA, E.; MERCANTE, F.M.; FRANCO, A.A.; GRAHAM, P. H. \& PARDO, M.A. Rhizobium tropici, a novel species nodulating Phaseolus vulgaris L. beans and Leucaena sp. trees. Int. J. Syst. Bacteriol., 41:417-426, 1991.

MERCANTE, F.M. Uso de Leucaena leucocephala na obtenção de Rhizobium tolerante a temperatura elevada para inoculação do feijoeiro. Seropédica, Universidade Federal Rural do Rio de Janeiro, 1993. 149p. (Tese de Mestrado)

MERCANTE, F.M.; TEIXEIRA, M.G.; ABBOUD, A.C.S. \& FRANCO, A.A. Avanços biotecnológicos na cultura do feijoeiro sob condições simbióticas. R. Univ. Rural: Sér. Ciênc. Vida, 21:127-146, 1999.

MERCANTE, F.M.; OTSUBO, A.A. \& LAMAS, F.M. Inoculação de Rhizobium tropici e aplicação de adubo nitrogenado na cultura do feijoeiro. In: REUNIÃO BRASILEIRA DE FERTILIDADE DE SOLO E NUTRIÇÃO DE PLANTAS, 27.; REUNIÃO BRASILEIRA SOBRE MICORRIZAS,11.; SIMPÓSIO BRASILEIRO DE MICROBIOLOGIA DO SOLO, 9.; REUNIÃO BRASILEIRA DE BIOLOGIA DO SOLO, 6., 2006, Bonito, MS. Fertbio 2006: A busca das raízes. Dourados, Embrapa Agropecuária Oeste, 2006. CD-ROM. (Documentos, 82)

PERES, J.R.R.; SUHET, A.R.; MENDES, I.C. \& VARGAS, M.A.T. Efeito da inoculação com rizóbio e da adubação nitrogenada em sete cultivares de feijão em solo de cerrado. R. Bras. Ci. Solo, 18:415-420, 1994.
SANTOS, A.B.; FAGERIA, N.K.; SILVA, O.F. \& MELO, M.L.B. Resposta do feijoeiro ao manejo de nitrogênio em várzeas tropicais. Pesq. Agropec. Bras., 38:1265-1271, 2003.

SILVA, F.A.S. \& AZEVEDO, C.A.V. Versão do programa computacional Assistat para o sistema operacional Windows. R. Bras. Prod. Agroind., 4:71-78, 2002.

SIQUEIRA, J.O.; MOREIRA, F.M.S.; GRISI, B.M.; HUNGRIA, M. \& ARAUJO, R.S. Microrganismos e processos biológicos do solo: Perspectiva ambiental. Santo Antônio de Goiás, Embrapa-CNPAF; Londrina, Embrapa-CNPSo; Brasília, Embrapa-SPI, 1994. p.47-50. (Documentos, 45)

SORATTO, R.P.; CARVALHO, M.A.C. \& ARF, O. Fertilidade do solo e nutrição de plantas. R. Bras. Ci. Solo, 30:259265,2006

SORATTO, R.P.; CRUSCIOL, C.A.C.; SILVA, L.M. \& LEMOS, L.B. Aplicação tardia de nitrogênio no feijoeiro em sistema de plantio direto. Bragantia, 64:211-218, 2005.

STRALIOTTO, R.; TEIXEIRA, M.G. \& MERCANTE, F.M. Fixação biológica de nitrogênio. In: AIDAR, H.; KLUTHCOUSKI, J. \& STONE, L.F. Produção de feijoeiro comum em várzeas tropicais. Santo Antônio de Goiás, Embrapa Arroz e Feijão, 2002. p.122-153.

YOKOYAMA, L.P. Aspectos conjunturais da produção de feijão. In: AIDAR, H.; KLUTHCOUSKI, J. \& STONE, L.F. Produção de feijoeiro comum em várzeas tropicais. Santo Antônio de Goiás, Embrapa Arroz e Feijão, 2002. p.249292. 